\title{
REVIEW
}

\section{Role of fibrin D-dimer testing in emergency medicine}

\author{
A Wakai, A Gleeson, D Winter
}

Emerg Med J 2003;20:319-325

See end of article for authors' affiliations .....................

Correspondence to: Dr A Wakai, Department of Emergency Medicine, Beaumont Hospital, Dublin 9, Ireland; wakai@indigo.ie

Accepted for publication 27 November 2002

\begin{abstract}
Objectives: Systemic values of the fibrinolytic plasma marker fibrin D-dimer are raised in a variety of acute clinical conditions. D-dimer values can now be rapidly determined and used to aid diagnosis in emergency medicine. However, despite clinical guidelines, inappropriate and unnecessary measurement of D-dimer values is a significant clinical problem. An understanding of the pathophysiological basis and limitations of the value of D-dimer values may help reduce this problem. This review discusses the pathophysiology of the fibrinolytic system. The currently used assays, clinical indications, and limitations of D-dimer measurement are reviewed. Finally, the potential future clinical indications for measurement of D-dimer values in emergency medicine are discussed.

Methods: Literature on D-dimer was identified from Medline, along with cross referencing from the reference lists of major articles on the subject

Results and conclusions: Systemic D-dimer values aids diagnosis, and is potentially a prognostic indicator, in a variety of clinical conditions in emergency medicine. However, it has limited specificity in patients with comorbid conditions. Although, currently, there is no standard D-dimer assay, immunoturbidimetric assays are the most suitable for use in emergency medicine
\end{abstract}

$\mathrm{T}$ he antigen fibrin D-dimer (DD) is the primary enzymatic degradation product of cross linked fibrin by plasmin. Systemic values of DD are an index of fibrin turnover in the circulation and a single measurement may be adequate to assess the fibrinolytic status. ${ }^{1}$ Systemic DD values are raised in a variety of clinical conditions ${ }^{2-7}$ and inclusion of DD testing may provide cost effective diagnostic strategies. ${ }^{8}{ }^{9}$ In addition to the diagnostic use of $\mathrm{DD}$, it may also be of potential prognostic use in many conditions. ${ }^{10-14}$ Currently, despite the implementation of clinical guidelines, inappropriate DD testing is a significant problem. ${ }^{15}$ It is, consequently, valuable for emergency physicians to be knowledgeable about the pathophysiological basis and limitations of DD testing to ensure its appropriate clinical use.

The place of DD measurement in the diagnostic investigation of suspected venous thromboembolism (VTE) is now well established. This review updates experience on DD testing for VTE and discusses potential areas for the extension of indications for DD testing in emergency medicine.

\section{PATHOPHYSIOLOGY OF FIBRIN DD}

Plasmin is the fibrinolytic enzyme derived from its inactive precursor, plasminogen, by the action of thrombin and plasminogen activators. The main plasminogen activators are tissue plasminogen activator (tPA) and pro-urokinase, which is activated into urokinase by, among others, the contact system of coagulation. ${ }^{16}$ Plasmin is neutralised by $\alpha_{2}$ antiplasmin thereby restricting its fibrinogenolytic activity and localising the fibrinolysis on the fibrin clot. ${ }^{16}$

Fibrin is the main component of a thrombus. It is formed by the activation of the coagulation system. Its production is followed by activation of the fibrinolytic system, resulting in plasmin generation and subsequent fibrin lysis (fig 1). Under normal physiological conditions there is a balance of the two opposing processes. Dissolution of crosslinked fibrin (XL-Fg) leads to formation of specific degradation products, including $\mathrm{DD},{ }^{17}$ which can be measured in both whole blood and plasma using monoclonal antibodies directed against epitopes located in the DD fragment. The activity of DD is considered to reflect the overall activity of clot formation and lysis. Because DD is not artificially generated in vitro during blood collection, its

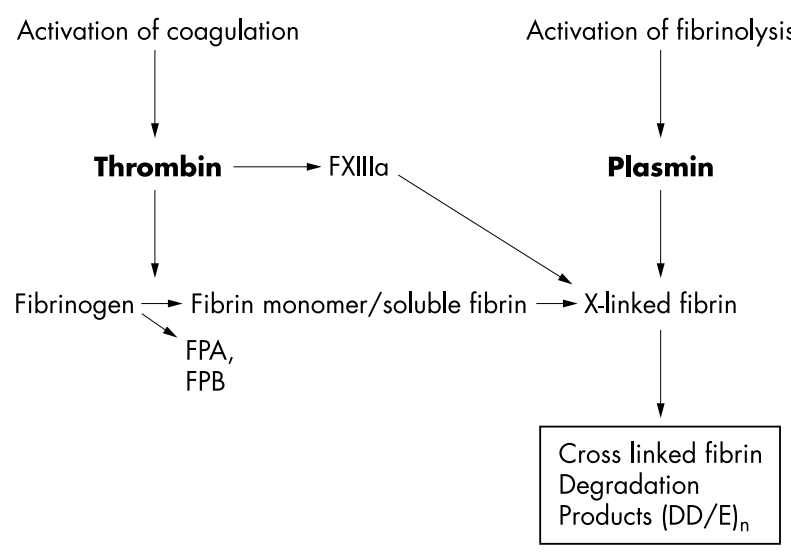

Figure 1 D-dimer as a reactive marker of the haemostatic balance. Activation of the coagulation system leads to the formation of thrombin. This enzyme cleaves the amino-terminal fibrinopeptides $A$ and $B$ from fibrinogen. The resulting fibrin monomer molecule is then capable to polymerise into an insloluble fibrin network. The fibrin poymers are further stabilised by covalent crosslinks introduced by the action of the enyzme factor XIlla. Plasmin, the active focal enzyme of the fibrinolytic sytem, is responsible for the lysis of the crosslinked fibrin clot. This results in the formation of soluble crosslinked FDPs containing a myriad of DD epitopes. (Adapted with permission from Houdijk WPM. Clinical applications of D-dimer. Dev Thromb Hemost 1999;3:1-15).

measurement more consistently reflects in vivo haemostatic activity than do other assays for coagulation or fibrinolytic activities that may be activated in vitro. Its absence excludes the presence of intravascular thrombus.

Abbreviations: DD, D-dimer; VTE, venous thromboembolism; tPA, tissue plasminogen activator; XL-Fg, crosslinked fibrin; FDP, fibrin degradation product; ELISA, enzyme linked immunosorbent assay; HMW, high molecular weight; LMW, low molecular weight; FACT, Fibrin Assay Comparison Trial; CT, computed tomography; PE, pulmonary embolism; DVT, deep vein thrombosis; PTP, pretest probability; CHD, coronary heart disease; ACS, acute coronary syndrome; MI, myocardial infarction 
Box 1 Conditions associated with increased DD titres

\section{Non-pathological}

- Cigarette smoking 22

- Age (healthy elderly people) 22

- Functional impairment ${ }^{23}$

- Race (black population $)^{23}$

- Pregnancy $y^{2}$

- Postoperatively 25

Pathological

- Trauma ${ }^{27}$

- Pre-eclampsia 2829

- Malignancy

- Infection ${ }^{30} 31$

- Disseminated intravascular coagulation ${ }^{32}$

- Sickle cell disease ${ }^{33}$

- Arterial or venous thromboembolism ${ }^{2-4}$

- Atrial fibrillation ${ }^{34}$

- Acute coronary syndromes ${ }^{35}$

- Stroke $36-38$

- Acute upper gastrointestinal haemorrhage ${ }^{14} 39$

During fibrin formation, fibrinogen is converted into fibrin by enzymatic (thrombin) cleavage of the fibrinopeptides $\mathrm{A}$ and B (fig l). This is followed by aggregation of the resulting fibrin monomers. Linkage of $\mathrm{C}$ terminal appendages of the $\gamma$ chains by factor XIIIa results in dimerisation of D-domains of adjacent fibrin monomer units. Plasmin proteolysis of this "crosslinked" fibrin generates fragments DD and E as terminal products. ${ }^{18}$ In contrast, proteolysis of fibrinogen or non-crosslinked fibrin (non-XL-Fg) results in formation of monomeric fragment $\mathrm{D}$. The dimeric D-domain therefore may serve as indicator of in vivo fibrin formation. ${ }^{19}$ Monoclonal antibodies generated by immunisation with fragment DD react with XL-Fg in general, including fibrin oligomers of variable size. $^{2021}$

DD testing has limited specificity because many conditions are associated with fibrin formation (box 1). Local fibrin formation and lysis are part of the inflammatory response, and fibrin degradation products (FDPs), including DD, have been shown to modulate acute phase responses and the production of a variety of systemic inflammatory mediators. ${ }^{40-42}$ Because of this low specificity, DD testing may not be helpful in hospitalised patients in whom comorbidity is common. ${ }^{43}{ }^{44}$ In addition, DD assays (especially qualitative latex agglutination assays) are not $100 \%$ sensitive, limiting their utility as a single screening test. ${ }^{43-50}$ Despite these limitations, there is mounting evidence that in the absence of an increase in DD, specifically by the more sensitive enzyme linked immunosorbent assay (ELISA), VTE is rare, especially if other non-invasive test results are negative or non-diagnostic. ${ }^{96}$ 50-61

\section{DD ASSAYS}

DD assays detect the presence in whole blood or plasma of plasmin mediated FDPs that contain cross linked D fragments. DD assays currently available for clinical use include: manual immunoagglutination assays, manual immunochromatographic assays, immunofiltration assays, microtitre plate ELISA) systems, automated ELISA systems, and latex enhanced photometric immunoassays. All these assays use a monoclonal antibody against epitopes on the DD fragment that are absent on fibrin, fibrinogen, and non-crosslinked fragments of fibrin. Depending on the degree of lysis of XL-Fg, a heterogeneous mixture of FDPs of different molecular weight containing the DD moiety may be formed. The reactivity of different DD monoclonal antibodies to these different molecular weight species varies, causing variable results with different assays within individual patients. In addition, some monoclonal antibodies may react with non-crosslinked degradation products of fibrin or fibrinogen. ${ }^{62}$
Box 2 Factors that underlie the discrepancy between DD assays

- Antibody specificity

- Time dependence of neo-epitome expression in the course of fibrin formation and dissolution

- Assay format

- Purity or heterogeneity of the calibrator

- Possible matrix effects of plasma on epitope presentation

- Interference by irrelevant (non-crosslinked) analytes

Rapid test results are mandatory in emergency medicine. Among them, a whole blood manual immunoagglutination test that gives a qualitative result in two minutes (SimpliRED, Agen Biochemical, Brisbane, Australia) and the more recent immunochromatographic test (Simplify, Agen Biochemical) that gives a semi-quantitative result in 10 minutes. Meanwhile a rapid plasma ELISA assay (VIDAS DD, bioMérieux UK, Basingstoke, UK) requires 35 minutes to produce a quantitative result. Unfortunately, immunoagglutination tests, including the whole blood SimpliRED test, have the disadvantages of visual interpretation (with potential interobserver variation) and limited sensitivity. On the other hand, the rapid ELISA test is highly sensitive, but is too cumbersome for emergency use. Despite the fact that ELISA has been considered the gold standard of DD assay methodology, new turbidimetric immunoassays may be equal in sensitivity and may even have a slightly better specificity in ED patients. ${ }^{63}$ Moreover, the turbidimetric assays are faster and are less cumbersome than ELISA. Among the currently available assays, the turbidimetric assays, are therefore, the best for routine use in emergency medicine.

\section{Limitations of DD assays}

DD tests are not directly comparable because they use different monoclonal antibodies and apply different units (for example, $\mathrm{ng} / \mathrm{ml}, \mu \mathrm{g} / \mathrm{ml}, \mathrm{mg} / \mathrm{ml}$, fibrinogen equivalent units (FEU) and DD units). Furthermore, they are often evaluated in different patient populations. For example, depending on the selection of patients studied, specificity varies from $24 \%$ to $82 \%$ using the same assay type. ${ }^{64}{ }^{65}$ Because of inter-assay and intra-assay variations and differences in patient populations, a wide range of sensitivities and specificities has been reported for DD assays. ${ }^{451}{ }^{66-68}$ The reported DD assay sensitivity and specificity varies so widely that its diagnostic utility has been questioned. ${ }^{45} 5166-68$ The reason for these differences between DD assays is multifactorial (box 2). Assay specific characteristics such as preference for high molecular weight (HMW) or low molecular weight (LMW) fibrin derivatives, or cross reactivity with non-crosslinked fibrinogen and fibrin degradation products should be taken into consideration upon interpretation of clinical DD assay results. ${ }^{69}$

Different calibrators are used for DD assays. Calibrators currently used include: terminal plasmin digest of XL-Fg clot, pooled plasma from patients with disseminated intravascular coagulation (DIC), and HMW-fibrin oligomer preparation. Manufacturers of DD assays have chosen their calibrators to suit the needs of their respective assay system. Consequently, there is no DD reference standard and inter-assay correlation of DD testing is, therefore, poor. This results in a rather arbitrary scale of the numerical results obtained. Although most DD assays are calibrated with terminal plasmin digests of XL-Fg clot, this calibrator generates aberrant results in some assays. ${ }^{69}$ Calibration with serial dilutions of pooled plasma from patients with DIC improves conformity between assays but has several drawbacks, including: variable composition of the biological material, unpredictable matrix effects, and the possibility of infections. ${ }^{69}$ Presently, the most suitable common calibrator is a standardised in vitro preparation with 
a composition of fibrin derivatives similar to that observed in the target clinical plasma samples, immersed in a plasma matrix. ${ }^{69}$

The lack of a DD reference standard can be overcome by conversion of DD values from different assays to a common scale by using a conversion factor related to the median values obtained with a sufficiently large set of clinical plasma samples. ${ }^{70}$ This issue has recently been investigated by the Fibrin Assay Comparison Trial (FACT) study. ${ }^{69}$ Briefly, the FACT study was performed to generate basic data for development of calibrators and standard preparations of DD. It is an attempt to propose a method of preparation of a universal set of DD calibrators that could potentially be used as reference materials. The difference observed in reactivity between different commercial kits occurred only with artificially created plasma samples and those of patients treated with streptokinase. ${ }^{69}$ True patient samples (DIC or deep venous thrombosis), as compared with standardised in vitro fibrin preparations, demonstrate a high degree of concordance by all the methods tested. ${ }^{69}$ This indicates that even if different DD assays respond to different epitopes, these epitopes are either present in parallel on the fibrin compounds detected, or fibrin compounds carrying the respective epitopes occur simultaneously in most clinical plasma samples. Differences concerning analyte reactivity will, therefore, become more evident only in samples containing an unusual composition of fibrin derivatives. Nevertheless, no definitive common scale for conversion of DD values from different assays has been formulated by the FACT study, so far. Meanwhile, this study suffers from two important limitations. Firstly, although a number of assays tested used the same antibodies, no attempt was made to classify the data according to the antibodies used. This automatically introduced bias to the results. Secondly, the study was based on a limited number of samples and the materials used for this testing were artificially modified plasma/serum samples created using a very specific procedure with degradation products not existing in vivo. Changing this procedure may significantly alter the results.

\section{DD assay cut off points}

Because different DD assays recognise more or less different components in plasma or in blood and because various techniques are used, heterogeneity of results is somewhat inevitable. Thus, rather than attempts at standardisation, some investigators suggest efforts should focus on determining a critical cut off for each individual assay, based on the test performances established in clinical studies. ${ }^{71}$ Cut off points are critical when using DD assays because they determine the sensitivity and specificity. If the DD cut off is set too low, then the test is too sensitive and not specific, so almost everyone ends up being positive and the test loses meaning. Laboratories can accept meaningful, published cut off points, but should not extrapolate a cut off point from the manufacturer of another assay. Furthermore, to ensure good applicability, when chosing a DD assay it should be verified that the assay has been studied in a patient population similar to that in which it would be used.

\section{CLINICAL APPLICATIONS OF DD TESTING Pulmonary embolism (PE)}

Despite the existence over the past decade of a decision rule for the evaluation of $\mathrm{PE}^{72}$ its diagnostic evaluation, more than any other acute clinical condition, continues to pose a dilemma for emergency physicians. ${ }^{73}$ A perfusion-ventilation (V/Q) lung scan followed by pulmonary angiography in conjunction with a low probability lung scan is generally considered to be the standard diagnostic strategy. ${ }^{72}$ Although a negative qualitative DD assay does not have the required sensitivity to rule out PE in the ED setting, ${ }^{75} \mathrm{DD}$ when measured with a quantitative assay, is nearly always increased in acute
PE (sensitivity 85\%-99\%), and a low value virtually rules it out. Furthermore, despite a low specificity $(45 \%-68 \%)$, diagnostic strategies incorporating DD testing are cost effective. $^{8} 97677$

Various strategies incorporating DD testing for emergency evaluation of PE have been proposed. These include using a rapid plasma ELISA DD as an initial screening test, ${ }^{51}$ using whole blood agglutination DD in combination with spiral computed tomography $(\mathrm{CT})^{77}$ and using whole blood agglutination DD in combination with pretest clinical probability (pretest probability; PTP) ${ }^{78}$ Nevertheless, there is no universal consensus on the role of DD testing in the emergency evaluation of PE.

A negative ELISA DD result as a single initial screening test, while simultaneously ignoring the PTP, safely eliminates a third of patients with suspected PE from further more expensive and invasive diagnostic tests. ${ }^{51}$ None the less, caution should be exercised with this approach, particularly in patients with a high PTP, until further confirmatory data are available. A more conservative approach would be to combine the results of the DD assay with PTP and other non-invasive diagnostic tests (duplex ultrasonography of lower limb veins and V/Q scanning). ${ }^{79}$ Diagnosis of lower limb deep venous thrombosis (DVT) as an alternative to proving the presence of $\mathrm{PE}$, among patients with suspected PE and inconclusive lung scans, is now a valid concept. ${ }^{89} \mathrm{~A}$ combination of DD measurement and lower limb venous ultrasonography can safely eliminate one third of patients with suspected PE. ${ }^{81}$

Although V/Q scanning is still widely used in many institutions as the definitive test for detecting PE, it is a poor test, providing a definitive clinical answer in only $27 \%$ of cases. ${ }^{72}$ In a prospective study, patients with suspected $\mathrm{PE}$ were randomised either to spiral CT or to V/Q scan as the initial screening test. ${ }^{82}$ Although there was no difference in the detection rate of emboli between the two groups, a confident diagnosis was possible with more patients in the CT group than in the group that underwent V/Q scanning. ${ }^{82}$ Furthermore, greater out of hours availability makes spiral CT a better primary screening test, than V/Q scanning, for ED patients with suspected PE. Meanwhile, incorporating DD testing (whole blood agglutination assay) is cheaper and further increases clinical efficiency, by reducing the number of spiral CT examinations in patients with suspected PE by $50 \%{ }^{77}$

DD testing (whole blood agglutination assay) in combination with a low PTP obviates the need for further investigation in $47 \%$ of ED patients with suspected PE. ${ }^{78}$ More importantly, outcome studies have demonstrated that it is safe to withhold further investigation and treatment in patients with a negative DD test, intermediate or low clinical suspicion for PE and a non-diagnostic (intermediate or low probability) V/Q scan. ${ }^{83} 84$

\section{Proximal acute lower limb DVT}

Duplex scanning, the present method of choice for the diagnosis of DVT, is comparatively time consuming and expensive. In addition, the majority of imaging studies for patients with suspected DVT, up to $98 \%$ in one study, are negative. ${ }^{85}$ A highly sensitive and simple test used for initial screening and ruling out DVT in a substantial proportion of subjects, might be quicker and cost effective. ${ }^{9}$ The more specific ultrasonographic test would then be applied only in patients presenting with a DD concentration above the appropriate cut off point. Although the precise role of DD tests in assessing suspected cases of DVT has yet to be established, it potentially meets these criteria.

Qualitative whole blood agglutination assays cannot be relied upon to exclude thrombosis in the initial assessment of suspected DVT. In one study, the diagnosis of DVT would have been missed in $20 \%$ of ED patients if only a whole blood agglutination DD assay was used. ${ }^{86}$ In contrast, quantitative 
assays can lead to a significant $(30 \%-50 \%)$ reduction in requests for imaging studies. ${ }^{85}{ }^{86}$ Meanwhile, a clinical scoring system can be used for risk stratification in DVT patients. ${ }^{87}$ This can be used in conjunction with ultrasound scanning and DD measurement in the work up of suspected DVT. ${ }^{61878990}$ If the clinical risk of DVT is low, the absence of thrombosis is supported by a negative qualitative whole blood DD test. ${ }^{61}{ }^{91}$

To date, the assays with the highest sensitivity in patients with suspected DVT are ELISA methods designed for batch analysis. These have a sensitivity between $97 \%-100 \%{ }^{45} 4692$ Although more sensitive than other DD assays, the applicability of ELISA DD assays in the ED is limited by the need for specialised equipment, multiple temperature and time dependent incubations and its laboratory turnaround time (about four hours). Consequently, less cumbersome and more rapid assays have been developed. The simplest include a whole blood agglutination assay (SimpliRED, Agen Biochemical) and, more recently, an immunochromatographic assay (Simplify, Agen Biochemical) These both give results within a few minutes and can be used at the bedside. ${ }^{78}{ }^{93}$ However, the diagnostic performance of the SimpliRED test in suspected DVT is highly variable with a sensitivity, specificity and negative predictive value of $61 \%-100 \%,{ }^{94} 58 \%-94 \%,{ }^{92}{ }^{96}$ and $52 \%-$ $100 \%,{ }^{45}{ }^{95} 96$ respectively. Although SimpliRED is less sensitive than ELISA assays, it seems adequate to safely rule out DVT in ED patients with a low PTP ${ }^{86}$ Moreover, it is more specific than the ELISA assays, obviating further investigation in $40 \%$ of patients with suspected DVT. ${ }^{61}$ Currently, there are limited published data on diagnostic performance of the Simplify DD assay besides that obtainable from the manufacturer. However, its interpretation is designed to be less susceptible to inter-observer variation than the SimpliRED assay, with visual interpretation of results very similar to that of a commercially available pregnancy test. In addition, it detects DD in both whole blood and plasma. In comparison with these assays based on visual interpretation of results, the more sensitive quantitative turbidimetric DD immunoassay used in conjunction with PTP is more reliable. ${ }^{86}$ The turbidimetric immunoassay takes about 20 minutes longer to perform than a whole blood assay but is not dependent on subjective interpretation. Furthermore, the turbidimetric immunoassay is more sensitive (94.1\% compared with $66 \%$ ) than the whole blood test and is $100 \%$ sensitive for proximal acute lower limb DVT. $^{86}$

\section{Calf vein acute DVT}

Although the clinical significance of calf vein thrombosis is controversial, ${ }^{97}$ many patients present to the ED specifically to clear the differential diagnosis of calf pain and rule out a DVT. Meanwhile, the risk of pulmonary embolism from isolated calf vein thrombosis seems negligible; however, a recurrence rate of $29 \%$ and propagation rate into more proximal veins of up to $28 \%$ have been reported. ${ }^{98-100}$ It is logical to hypothesise that patients with extending calf DVT have thrombi that are more active biochemically, more likely to produce increased plasma DD values, and therefore, more likely to show an abnormal DD assay result. ${ }^{101}$

In calf DVT, the qualitative whole blood agglutination DD assay has a moderate sensitivity $(70 \%)$, moderate specificity $(77 \%),{ }^{101}$ a false negative rate of $30 \%,{ }^{102}$ and NPV of only $85.7 \%{ }^{91}$ Meanwhile, a negative quantitative ELISA DD test with a negative compression sonogram of the popliteal and common femoral veins predicts the absence of a major thromboembolic event in the next three months in $97 \%$ of patients referred with suspicion of DVT..$^{51}$ However, because earlier studies have shown a substantial rate of progression of untreated calf DVT ${ }^{100} 103104$ it is, therefore, important to confirm every reasonable suspicion of thrombotic calf pain quickly and objectively with the aid of a quantitative DD assay.

\section{Recurrent VTE}

It may be very difficult to distinguish recurrent VTE from the sequelae of a previous event. DD estimation is potentially useful for this purpose because DD levels return to normal values within three months after an acute lower limb DVT. ${ }^{98}$ Thus, in a patient suspected of a recurrent VTE event, a low DD value in conjunction with clinical assessment and appropriate investigation could be used to rule out recurrence. ${ }^{105}$

\section{Coronary ischaemia}

DD is positively associated with coronary heart disease (CHD) incidence $^{22106}$ or recurrence. ${ }^{13}$ This association is largely independent of classic risk factors such as smoking and hypercholesterolaemia. ${ }^{6}$ CHD risk is about $70 \%$ greater in those in the top third of DD values compared with those in the bottom third. ${ }^{13}$ 107-111

Meanwhile, there remains a need for additional markers in the early stages of coronary thrombosis, a hallmark of acute ischaemic syndromes, ${ }^{112}$ because a persistent $2 \%$ to $4 \%$ of patients discharged from the ED may have a myocardial infarction (MI) within 24-48 hours with current diagnostic tools. ${ }^{13}$ Bayes-Genis et al have recently demonstrated that plasma DD values are significantly higher in patients with acute ischaemic events (MI and unstable angina) than in non-ischaemic patients. ${ }^{35}$ Moreover, plasma DD values for MI are significantly higher than for unstable angina. ${ }^{35} \mathrm{DD}$ concentration $>500 \mu \mathrm{g} / \mathrm{l}$ has an independent diagnostic value for MI and increases the diagnostic sensitivity of the electrocardiogram and history from $73 \%$ to $92 \% .{ }^{35}$

Progression of coronary thrombosis may be an important determinant of prognosis in patients with acute coronary syndromes (ACS), judging from the decrease in recurrent ischaemia, infarction and death associated with antithrombotic and antifibrinolytic therapy. ${ }^{114}{ }^{115}$ Identification of patients at risk of progression of coronary thrombosis has only been possible by coronary angiography, with no clinically applicable laboratory assays to characterise the balance between thrombosis and fibrinolysis. Angiography is an insensitive method of detection of coronary thrombosis when compared with direct thrombus visualisation by angioscopy, an equally invasive procedure. ${ }^{116} \mathrm{DD}$ assays may permit rapid bedside risk stratification of patients with ACS who are at higher risk of thrombotic complications. ${ }^{116}$

Plasma DD value is a potentially useful marker of CHD risk, ${ }^{22}{ }^{106}$ an addition to clinical diagnostic models for MI detection in ED patients with acute cardiogenic chest pain ${ }^{35}$ and may identify ACS patients who may benefit from more aggressive antithrombotic therapy. ${ }^{116}$ Currently the use of DD testing in ACS is limited by the inherent lack of specificity of most assays for the detection of coronary thrombosis. This limitation may at least be partially obviated using diagnostic strategies incorporating other more established markers of myocardial damage such as cardiac specific troponins. ${ }^{116}$

\section{DD AS AN ED PROGNOSTIC MARKER}

As clinical decision units and more prolonged periods of treatment become more common in the ED, there is need for rapid bedside adjunctive diagnostic aids in risk stratification of the ED patient population. In addition to ACS, DD is potentially useful as a prognostic marker in conditions such as acute bowel ischaemia, ${ }^{4}$ acute upper gastrointestinal haemorrhage, ${ }^{149}$ intracranial haemorrhage, ${ }^{3738}$ cerebral infarction, ${ }^{36}$ atrial fibrillation, ${ }^{34}$ and bacteraemia. ${ }^{7}$

Increased plasma DD is associated with adverse clinical outcome in acute upper gastrointestinal haemorrhage ${ }^{14}$ and intracranial haemorrhage. ${ }^{37} \mathrm{DD}$ values may be an indicator for emergency laparotomy in acute bowel ischaemia ${ }^{4}$ and emergency endoscopy in upper gastrointestinal haemorrhage. ${ }^{14}$ In cerebral infarction, DD values may indicate the aetiology. Cardioembolic (diagnosed in the presence of a 
detectable cardiac embolic source without large vessel disease) and atherothrombotic (diagnosed in the presence of large vessel disease without a cardiac embolic source) strokes are associated with significantly increased DD values. ${ }^{117}$ In contrast, in lacunar stroke (small subcortical infarcts without a cardiac source or large vessel disease) DD values are not significantly changed. ${ }^{117}$

Clinical applicability of current data regarding the prognostic role of DD suffers from several limitations including studies that are retrospective, ${ }^{34}$ have small sample sizes, ${ }^{4734}$ and use qualitative rather than quantitative DD assays. $^{7}$ In addition, some studies use the DVT cut off value for diagnosis. ${ }^{4}$ The DVT cut off value may not be scientifically valid in the diagnosis of other clinical conditions. Larger prospective studies, using quantitative DD assays with appropriate cut off points, in the ED setting are required before DD values can be routinely used for risk stratification of patients in the ED setting.

\section{CONCLUSION}

Measurement of systemic DD, an index of ongoing thrombus formation and lysis, can aid clinical diagnosis in venous thromboembolic conditions and acute coronary syndromes. In addition, it may potentially permit risk stratification in the ED of patients with calf DVT, acute upper gastrointestinal haemorrhage, intracranial haemorrhage, and bacteraemia, thereby permitting a targeted approach to further investigation and treatment.

DD has limited specificity in hospitalised patients where comorbidity is common. In ED patients, there is mounting evidence that in the absence of an increase in $\mathrm{DD}$, specifically in patients with low PTP, venous thromboembolism is rare, particularly if other non-invasive tests are negative or equivocal.

Although ELISA DD assay is the gold standard, it is too slow and cumbersome for use in emergency medicine. Meanwhile, the diagnostic performance of the less cumbersome and comparatively quick immunoturbidimetric assays compare favourably with ELISA. Turbidimetric immunoassays are therefore currently the best DD assay for use in emergency medicine.

Attempts to standardise the various DD assays is ongoing with no definitive answers yet. In the meantime, DD assays should be used in conjunction with clinical assessment. Cut off points and the patient population used in preparing the assay should also be taken into consideration upon interpretation of assay results.

Before definitive incorporation of DD into diagnostic clinical models in emergency medicine, more studies using quantitative DD assays are required to further refine current knowledge, which is largely based on qualitative assays.

\section{ACKNOWLEDGEMENTS}

The authors thank Geraldine Healy, Haematology Laboratory, Beaumont Hospital, for reading the manuscript and providing some useful references.

\section{Contributors}

Abel Wakai and Aidan Gleeson initiated the review. Abel Wakai, Aidan Gleeson and Desmond Winter produced the final manuscript. Abel Wakai acts as the guarantor for the paper.

\section{Authors' affiliations}

A Wakai, A Gleeson, Department of Emergency Medicine, Beaumont Hospital, Dublin, Republic of Ireland

D Winter, Department of Surgery, Beaumont Hospital

\section{REFERENCES}

1 Rao KMK, Pieper C, Currie MS, et al. Variability of plasma IL-6 and crosslinked fibrin dimers over time in community dwelling elderly subjects. Am J Clin Pathol 1994; 102:802-5.
2 Harrison KA, Haire WD, Pappas AA, et al. Plasma D-Dimer: a useful tool for evaluating suspected pulmonary embolus. J Nucl Med 1993;34:896-8

3 Meissner MH, Zierler BK, Bergelin RO, et al. Markers of plasma coagulation and fibrinolysis after acute deep venous thrombosis. J Vasc Surg 2000;32:870-80

4 Acosta S, Nilsson TK, Björck M. Preliminary study of D-dimer as a possible marker of acute bowel ischaemia. Br J Surg 2001;88:385-8. 5 Yukihiko F, Takeuchi S, Harada A, et al. Hemostatic activation in spontaneous intracerebral hemorrhage. Stroke 2001;32:883-90.

6 Danesh J, Whincup P, Walker M, et al. Fibrin D-dimer and coronary heart disease. Circulation 2001;103:2323-7.

7 Quick G, Eisenberg P. Bedside measurement of D-dimer in the identification of bacteremia in the emergency department. J Emerg Med 2000; 19:217-23.

8 Michel BC, Seerden RJ, Rutten FH. The cost-effectiveness of diagnostic strategies in patients with suspected pulmonary embolism. Health Econ 1996;5:307-18

9 Perrier A, Buswell L, Bounameaux $\mathrm{H}$, et al. Non-invasive diagnostic aids are cost-effective in suspected pulmonary embolism. Arch Intern Med 1997; 157:2309-16.

10 Lip GYH, Rumley A, Dunn FG, et al. Plasma fibrinogen and fibrin D-dimer in patients with atrial fibrillation. Int J Cardiol 1995;51:245-51.

11 Lip GYH, Zafiris J, Watson RDS, et al. Fibrin D-dimer and $\beta$-thromboglobulin as markers of thrombogenesis and platelet activation in atrial fibrillation: effects of introducing ultra-low-dose warfarin and aspirin. Circulation 1996;94:425-31.

12 Lowe GDO, Rumley A. Use of fibrinogen and fibrin D-dimer in prediction of arterial thrombotic events. Thromb Haemost 1999;82:667-72.

13 Moss AJ, Goldstein RE, Marder VJ, et al. Thrombogenic factors and recurrent coronary events. Circulation 1999;99:2517-22.

14 Gutiérrez A, Sánchez-Payá J, Marco P, et al. Prognostic value of fibrinolytic tests for hospital outcome in patients with acute upper gastrointestinal hemorrhage. J Clin Gastroenterol 2001;32:315-18.

15 Durieux P, Dhôte P, Meyniard O, et al. D-dimer testing as the initial test for suspected pulmonary embolism. Appropriateness of prescription and physician compliance to guidelines. Thromb Res 2001;101:261-6.

16 Bachmann F. Fibrinolysis. In: Verstraete M, Vermylen J, Lijnen HR, et al, eds. Thrombosis and haemostasis. Leuven: Leuven University Press, 1987:227-65

17 Kroneman R, Nieuwenhuizen W, Knot EAR. Monoclonal antibody-based plasma assays for fibrin(ogen) and derivatives, and their clinical relevance. Blood Coagul Fibrinolysis 1990;1:91-111.

18 Marder VJ, Francis CW. Plasmin degradation of cross-linked fibrin. Ann NY Acad Sci 1983;408:397-406.

19 Whitaker AN, Rowe EA, Masci PP, et al. Identification of D-dimer-E-complex in disseminated intravascular coagulation. Thromb Res 1980; 18:453-9.

20 Brenner B, Francis CW, Marder VJ. The role of soluble cross-linked fibrin in D dimmer immunoreactivity of plasmic digests. J Lab Clin Med 1989:113:682-8.

21 Pfitzner SA, Dempfle CE, Heene DL. Fibrin detected in plasma of patients with disseminated intravascular coagulation by fibrin-specific antibodies consists primarily of high molecular weight factor XIIla-crosslinked and plasmin-modified complexes partially containing fibrinopeptide A. Thromb Haemost 1997;78: 1069-78.

22 Lowe GDO, Yarnell JW, Rumley A, et al. C-reactive protein, fibrin D-dimer, and incident ischemic heart disease in the Speedwell Study: are inflammation and fibrin turnover linked in pathogenesis? Arterioscle Thromb Vasc Biol 2001;21:603-10.

23 Pieper CF, Rao KM, Currie MS, et al. Age, functional status, and racial differences in plasma D-dimer levels in community-dwelling elderly persons. J Gerontol Med Sci 2000;55:M649-57.

24 Giavarina D, Mezzena G, Dorizzi RM, et al. Reference interval of D-dimer in pregnant women. Clin Biochem 2001;34:331-3.

25 Larsen JF, Ejstrud P, Svendsen F, et al. Randomized study of coagulation and fibrinolysis during and after gasless and conventional laparoscopic cholecystectomy. Br J Surg 2001;88:1001-5.

26 Nguyen NT, Owings JT, Gosselin R, et al. Systemic coagulation and fibrinolysis after laparoscopic and open gastric bypass. Arch Surg 2001;136:909-16.

27 Engelman DT, Gabram SGA, Allen L, et al. Hypercoagulability following multiple trauma. World J Surg 1996;20:5-10.

28 Kobayashi T, Tokunaga N, Sugimura $M$, et al. Coagulation/fibrinolysis disorder in patients with severe preeclampsia. Semin Thromb Hemost 1999:25:451-4.

29 Kobayashi T, Tokunaga N, Sugimura $M$, et al. Predictive values of coagulation/fibrinolysis parameters for the termination of pregnancy complicated by severe preeclampsia. Semin Thromb Hemost 2001;27:137-41.

30 Raimondi P, Bongard O, de Moerloose P, et al. D-dimer plasma concentration in various clinical conditions: Implication for the use of this test in the diagnostic approach of venous thromboembolism. Thromb Res 1993;69:125-30.

31 Jimenez Castro D, Perez-Rodriguez E, Montaner L, et al. Diagnostic value of $\mathrm{D}$-dimer in pulmonary embolism and pneumonia. Respiration 2001;68:371-5

32 Lane DA, Preston FE, Van Ross ME, et al. Characterization of serum fibrinogen and fibrin fragments produced during disseminated intravascular coagulation. Br J Haematol 1978:40:609-15.

33 Tomer A, Harker LA, Kasey S, et al. Thrombogenesis in sickle cell disease. J Lab Clin Med 2001;137:398-407. 
34 Nakagawa K, Hirai T, Shinokawa N, et al. Relation of fibrillatory wave amplitude with hemostatic abnormality and left atrial appendage dysfunction in patients with chronic nonrheumatic atrial fibrillation. Jpn Circ J 2001;101:13-21

35 Bayes-Genis A, Mateo J, Santalo M, et al. D-Dimer is an early diagnostic marker of coronary ischemia in patients with chest pain. Am Heart J 2000;140:379-84.

36 Berge E, Friis P, Sandset PM. Hemostatic activation in acute ischemic stroke. Thromb Res 2001;101:13-21.

37 Nina P, Schisano G, Chiapetta F, et al. A study of blood coagulation and fibrinolytic system in spontaneous hemorrhage. Correlation with Hunt-Hess grade and outcome. Surg Neurol 2001;55:197-203.

38 Fujii $Y$, Takeuchi S, Harada A, et al. Hemostatic activation in spontaneous intracerebral hemorrhage. Stroke 2001;32:883-90.

39 Vreeburg EM, Levi M, Rauws EA, et al. Enhanced mucosal fibrinolytic activity in gastroduodenal ulcer hemorrhage and the beneficial effect of acid suppression. Aliment Pharmacol Ther 2001;15:639-46.

40 Ritchie DG, Levy BA, Adams MA, et al. Regulation of fibrinogen and fibrin: an indirect feedback pathway. Proc Natl Acad Sci USA 1982;79: 1530-4.

41 Edgington TS, Curtiss LK, Plow EG. A linkage between the haemostatic and immune systems embodied in the fibrinolytic release of lymphocyte suppressive peptides. J Immunol 1985;134:471-7.

42 Gauldie J, Northemann W, Fey GHO. IL-6 functions as an exocrine hormone in inflammation: hepatocytes undergoing acute phase responses require exogenous IL-6. J Immunol 1990;144:3804-8.

43 Miron MJ, Perrier A, Bounameaux $\mathrm{H}$, et al. Contribution of non-invasive evaluation to the diagnosis of pulmonary embolism in hospitalised patients. Eur Respir J 1999:13:1365-70.

44 Freyburger G, Trillaud H, Labrouche S, et al. Rapid ELISA D-dimer testing in the exclusion of venous thromboembolism in hospitalised patients. Clin Appl Thromb Hemost 2000;6:77-81

45 Janssen MC, Heebels $A E$, de Metz M, et al. Reliability of five rapid d-dimer assays compared to ELISA in the exclusion of deep venous thrombosis. Thromb Haemost 1997:77:262-6.

46 Freyburger G, Trillaud H, Labrouche S, et al. D-dimer strategy in thrombosis exclusion - a gold standard study in 100 patients suspected of deep venous thrombosis or pulmonary embolism: $8 \mathrm{DD}$ methods compared. Thromb Haemost 1998;79:32-7.

47 Reber G, de Moerloose P, Coquoz C, et al. Comparison of two rapid D-dimer assays for the exclusion of venous thromboembolism. Blood Coagul Fibrinolysis 1998;9:387-8

48 Lee AY, Julian JA, Levine MN, et al. Clinical utility of a rapid whole-blood D-dimer assay in patients with cancer who present with suspected acute deep venous thrombosis. Ann Intern Med 1999:131:417-23.

49 Kutinsky I, Blakeley S, Roche V. Normal D-dimer levels in patients with pulmonary embolism. Arch Intern Med 1999;159:1569-72.

50 Farrell S, Hayes T, Shaw M. A normal SimpliRED D-dimer assay does not exclude the diagnosis of deep vein thrombosis or pulmonary embolism in emergency department patients. Ann Emerg Med 2000;35: 121-5

51 Perrier A, Desmarais S, Miron M, et al. Non-invasive diagnosis of venous thromboembolism in outpatients. Lancet 1999;353:190-5

52 Bounameaux H, Cirafici P, de Moerloose P, et al. Measurement of D-dimer in plasma as diagnostic aid in suspected pulmonary embolism. Lancet 1991;337:196-200.

53 De Moerloose $\mathbf{P}$, Desmarais S, Bounameaux H, et al. Contribution of a new, rapid, individual and quantitative automated D-dimer ELISA to exclude pulmonary embolism. Thromb Haemost 1996;75:1 1-13.

54 De Moerloose P, Michiels JJ, Bounameaux H. The place of D-dimer testing in an integrated approach of patients suspected of pulmonary embolism. Semin Thromb Haemost 1998;24:409-12.

55 Perrier A, Bounameaux H, Morabia A, et al. Diagnosis of pulmonary embolism by a decision analysis-based strategy including clinical probability, D-dimer levels, and ultrasonography: a management study. Arch Intern Med 1996;156:531-6.

56 Heit JA, Minor TA, Andrews JC, et al. Determinants of plasma fibrin D-dimer sensitivity for acute pulmonary embolism as defined by pulmonary angiography. Arch Pathol Lab Med 1999:123:235-40.

57 Janssen MC, Wollersheim H, Verbruggen B, et al. Rapid D-dimer assays exclude deep venous thrombosis and pulmonary embolism: current status and new developments. Semin Thromb Hemost 1998;24:393-400.

58 Oger E, Leroyer C, Bressollette L, et al. Evaluation of a new, rapid, and quantitative D-Dimer test in patients with suspected pulmonary embolism. Am J Respir Crit Care Med 1998;158:65-70.

59 Tardy B, Tardy-Poncet B, Viallon A, et al. Evaluation of D-dimer ELISA test in elderly patients with suspected pulmonary embolism. Thromb Haemost 1998;79:38-41

60 D'Angelo A, D'Alessandro G, Tomassini L, et al. Evaluation of a new rapid quantitative D-dimer assay in patients with clinically suspected deep vein thrombosis. Thromb Haemost 1996;75:412-16.

61 Kearon C, Ginsberg JS, Douketis J, et al. Management of suspected deep venous thrombosis in outpatients by using clinical assessment and D-dimer testing. Ann Intern Med 2001;135:108-11.

62 Eisenberg PR, Jaffe AS, Stump DC, et al. Validity of enzyme-linked immunosorbent assays of cross-linked fibrin degradation products as a measure of clot lysis. Circulation 1990;82:1159-68

63 Shitrit D, Heyd J, Raveh D, et al. Diagnostic value of the d-dimer test in deep vein thrombosis: improved results by a new assay method and by using discriminate levels. Thromb Res 2001;102:125-31.

64 Elias A, Aptel I, Huc B, et al. D-dimer test and diagnosis of deep vein thrombosis: a comparative study of 7 assays. Thromb Haemost 1996;76:518-22.
65 Legnani C, Pancani C, Palareti G et al. Contribution of a new, rapid quantitative and automated method of D-dimer measurement to exclude deep vein thrombosis in symptomatic outpatients. Blood Coagul Fibrinolysis 1999; 10:69-74.

66 Bounameaux H, de Moerloose P, Perrier A, et al. Plasma measurement of D-dimer as diagnosing aid in suspected venous thromboembolism: overview. Thromb Haemost 1994;71:1-6.

67 Kenecht $M$, Heinrich F. D-dimer assay in the diagnostic procedure of deep vein thrombosis and pulmonary embolism. Thromb Res 1997;88:413-17.

68 Ginsberg JS, Wells PS, Brill-Erlwards P, et al. Application of a novel and rapid whole blood assay for D-dimer in patients with clinically suspected pulmonary embolism. Thromb Haemost 1995;73:35-8.

69 Dempfle CE, Zips S, Ergul H, et al. The Fibrin Assay Comparison Tria (FACT): evaluation of 23 quantitative D-dimer assays as basis for the development of D-dimer calibrators. FACT Study Group. Thromb Haemost 2001:85:671-8.

70 Nieuwenhuizen $\mathbf{W}$. A reference material for harmonisation of D-dimer assays. Fibrinogen Subcommittee of the Scientific and Standardization Committee of the International Society of Thrombosis and Haemostasis. Thromb Haemost 1997:77: 1031-3.

71 Bounameaux H, DeMoerloose P, Perrier A, et al. D-Dimer testing in suspected venous thromboembolism: an update. QJM 1997;90:437-42.

72 The PIOPED Investigators. Value of the ventilation/perfusion scan in acute pulmonary embolism. Results of the prospective investigation of pulmonary embolism diagnosis (PIOPED). JAMA 1990:263:2753-9.

73 Graham I, Stiell IG, McAuley L, et al. Potential areas for new clinical decision rules: comparison of North America and Europe. [Abstract]. Acad Emerg Med 1999;6:433

74 Moser KM. Venous thromboembolism. Am Rev Respir Dis 1990;141:235-49.

75 Jones S, Harrison M. Towards evidence based medicine: best BETs from the Manchester Royal Infirmary. SimpliRed and diagnosis of deep venous thrombosis. Emerg Med J 2001;18:120-2.

76 Van Erkel AR, Van Rossum AB, Bloem JL, et al. Spiral CT angiography for suspected pulmonary embolism: a cost-effectiveness analysis. Radiology 1996;201:29-36.

77 Adams FG. The role of spiral computed tomography and D-dimer in pulmonary embolism. Scott Med J 2001;46:7-8

78 Wells PS, Anderson DR, Rodger $M$, et al. Excluding pulmonary embolism at the bedside without diagnostic imaging: management of patients with suspected pulmonary embolism presenting to the emergency department by using a simple clinical model and d-dimer. Ann Intern Med 2001;135:98-107

79 Wolfe TR, Hartsell SC. Pulmonary embolism: making sense of the diagnostic evaluation. Ann Emerg Med 2001;37:504-14.

80 Hull MD, Gary E, Raskob GE, et al. A new noninvasive management strategy for patients with suspected pulmonary embolism. Arch Intern Med 1989; 149:2549-55

81 Perrier A, Bounameaux H, Morabia A, et al. Contribution of D-dimer plasma measurement and lower-limb venous ultrasound to the diagnosis of pulmonary embolism: a decision analysis model Am Heart J 1994; 127:624-35

82 Cross JJ, Kemp PM, Walsh CG, et al. A randomized trial of spiral CT and ventilation perfusion scintigraphy for the diagnosis of pulmonary embolism. Clin Radiol 1998;53:177-82.

83 Perrier A, Desmarais S, Goehring C, et al. D-dimer testing for suspected pulmonary embolism in outpatients. Am J Respir Crit Care Med 1997; 156:492-6

84 de Groot MR, van Marwijk, Kooy M, et al. The use of a rapid D-dime blood test in the diagnostic work up of pulmonary embolism. Thromb Haemost 1999;82: 1588-92

85 Bucek RA, Koca N, Reiter M, et al. Algorithms for the diagnosis of deep-vein thrombosis in patients with low clinical pretest probability. Thromb Res 2002; 105:43-7.

86 Dryjski M, O'Brien-Irr MS, Harris LM, et al. Evaluation of screening protocol to exclude the diagnosis of deep venous thrombosis among emergency department patients. J Vasc Surg 2001;34:1010-15.

87 Wells PS, Anderson DR, Bormanis J, et al. Value of assessment of pretest probability of deep-vein thrombosis in clinical management. Lancet $1997 \cdot 350: 1795-8$

88 Harper P, Marson C, Grimmer A, et al. The rapid whole blood agglutination d-dimer has poor sensitivity for use as an exclusion test in suspected deep vein thrombosis. NZ Med J 2001;114:61-4.

89 Bernadi E, Prandoni P, Lensing AW, et al. D-dimer testing as an adjunct to ultrasonography in patients with clinically suspected deep vein thrombosis: prospective cohort study. The Multicentre Italian D-dimer Ultrasound Study Investigators Group. BM 1998;317:1037-40.

90 Lennox AF, Delis KT, Serunkuma S, et al. Combination of a clinical risk assessment score and rapid whole blood d-dimer testing in the diagnosis of deep vein thrombosis in symptomatic patients. J Vasc Surg 1999:30:794-803

91 Wells PS, Anderson DR, Bormanis J, et al. SimpliRED D-dimer can reduce the diagnostic tests in suspected deep vein thrombois. Lancet 1998;351:1405-6.

92 van der Graaf F, Van den Borne H, Van der Kolk M, et al. Exclusion of deep venous thrombosis with D-dimer testing. Comparison of $13 \mathrm{D}$-dimer methods in 99 outpatients suspected of venous thrombosis using venography as reference standard. Thromb Haemost 2000;83:191-8.

93 John MA, Elms M, O'Reilly EJ, et al. The simpliRED D-dimer test: a nove assay for the detection of crosslinked fibrin degradation products in whole blood. Thromb Res 1990;58:273-81. 
94 Mauron T, Baumgartner I, Z'Brun A, et al. SimpliRED D-dimer assay: comparability of capillary and citrated venous whole blood, between-assay variability, and performance of the test for exclusion of deep vein thrombosis in symptomatic outpatients. Thromb Haemost 1998:79:1217-19.

95 Mayer W, Hirschwehr R, Hippmann G, et al. Whole-blood immunoassay (SimpliRED) versus plasma immunoassay (NycoCard) for the diagnosis of clinically suspected deep vein thrombosis. Vasa 1997;26:97-101.

96 Turkstra F, van Beek EJ, ten Cate JW, et al. Reliable rapid blood test for the exclusion of venous thromboembolism in symptomatic outpatients. Thromb Haemost 1996;76:9-11.

97 Gottlieb RH, Widjaja J, Tian L, et al. Calf sonography for detecting deep venous thrombosis in symptomatic patients: experience and review of the literature. J Clin Ultrasound 1999;27:414-20.

98 Lagerstedt $\mathrm{Cl}$, Olsson CG, Fagher BO, et al. Need for long-term anticoagulant treatment in symptomatic calf-vein thrombosis. Lancet 1985; ii:515-18

99 Philbrick JT, Becker DM. Calf deep venous thrombosis. A wolf in sheep's clothing? Arch Intern Med 1988;148:2131-8.

100 Lohr JM, James KV, Deshmukh RM, et al. Calf vein thrombi are not a benign finding. Am J Surg 1995;170:86-90.

101 Wells PS, Brill-Edwards P, Stevens P, et al. A novel and rapid whole-blood assay for D-dimer in patients with clinically suspected deep vein thrombosis. Circulation 1995;91:2184-7.

102 Blättler W, Kneis N, Blättler IK. Practicability and quality of outpatient management of acute deep venous thrombosis. J Vasc Surg 2000;32:870-80.

103 Lindhagen A, Bergqvist D, Hallböök T, et al. Venous function five to eight years after clinically suspected deep venous thrombosis. Acta Med Scand 1985;217389-95.

104 Belcaro G, Laurora G, Cesarone MR, et al. Prevenzione della estensione della thrombosi venosa profonda distale. Minerva Med 1997;88:507-14.

105 Sié P, Cadroy Y, Elias A, et al. D-dimer levels in patients with long-term antecedents of deep venous thrombosis. Thromb Haemost 1994;72:161-2.
106 Folsom AR, Aleksic N, Park E, et al. Prospective study of fibrinolytic factors and incident coronary heart disease: the Atherosclerosis Risk in Communities (ARIC) Study. Arterioscler Thromb Vasc Biol 2001;21:611-17

107 Ridker PM, Hennekens $\mathrm{CH}$, Cerskus A, et al. Plasma concentration of cross-linked fibrin degradation product (D-dimer) and the risk of future myocardial infarction among apparently healthy men. Circulation 1994;90:2236-40

108 Smith FB, Lee AJ, Fowkes FGR, et al. Hemostatic factors as predictors of ischemic heart disease and stroke in the Edinburgh Artery Study. Arterioscler Thromb Vasc Biol 1997; 17:3321-5.

109 Lowe GDO, Yarnell JWG, Sweetnam PM, et al. Fibrin D-dimer, tissue plasminogen activator, plasminogen activator inhibitor, and the risk of major ischaemic heart disease in the Caerphilly study. Thromb Haemost 1998;79:129-33.

110 Cushman $M$, Lemaitre R, Kuller L, et al. Fibrinolytic activation markers predict myocardial infarction in the elderly: the cardiovascular health study. Arterioscler Thromb Vasc Biol 1999;19:493-8.

111 Smith FB, Rumley A, Lee AJ, et al. Haemostatic factors and prediction of ischaemic heart disease and stroke in claudicants. Br J Haematol 1998; 100:758-63.

112 Fuster V, Badimon L, Badimon JJ, et al. The pathogenesis of coronary artery disease and the acute coronary syndromes. N Engl J Med 1992;326:310-18.

113 Puleo PR, Meyer D, Wathen C, et al. Use of a rapid assay of subforms of creatine kinase $M B$ to diagnose or rule out acute myocardial infarction. N Engl J Med 1994;331:561-6.

114 Wallentin L. Low molecular weight heparins: a valuable tool in the treatment of acute coronary syndromes. Eur Heart J 1996;17:1470-6.

115 Van de Werf F. Clinical trials with glycoprotein Ilb/llla receptor antagonists in acute coronary syndromes. Thromb Haemost 1997;78:210-13.

116 Ottani F, Galvani M. Prognostic role of hemostatic markers in acute coronary syndrome patients. Clin Chim Acta 2001;311:33-9.

117 Uchiyama S, Yamazaki M, Hara Y, et al. Alterations of platelet, coagulation, and fibrinolysis markers in patients with acute ischemic stroke. Semin Thromb Haemostat 1997;23:535-41. 